\title{
NOVEL SOLUTION FOR LEG MOTION WITH 5-LINK BELT MECHANISM
}

\author{
E.Ch. LOVASZ*, C. POP, F. POP and V. DOLGA \\ Department of Mechatronics, Faculty of Mechanical Engineering \\ Politehnica University of Timisoara, ROMANIA \\ E-mails: erwin.lovasz@upt.ro, florina.pop@upt.ro, \\ cristian.pop@upt.ro, valer.dolga@upt.ro
}

\begin{abstract}
From the analysis of Theo Jansen walking mechanism and of the path curve that it describes the reduced capability for crossing over obstacles of the Jansen leg ( 1 DOF) is pointed out. By using a 5 link belt mechanism with 2 DOF can be adapted for generating similar Jansen mechanism path curve, where the step height of this path can be increased. For this purpose a mathematical model is conceived in order to analyse and determine the parameters for driving and control of the operation of the novel walking leg solution.
\end{abstract}

Key words: walking leg, Jansen leg, 5-link belt mechanism, path generation, kinematic analysis.

\section{Introduction}

The variety of walking robots prototypes that are developed around the world for different activities in domains like civil, military research, agriculture and entertainment are based on different types of leg configurations. The leg mechanism used as support for obtaining certain mobility for the walking robots represents the main objective for optimal construction analysis offering a series of advantages regarding manoeuvrability, static and dynamic stability.

Almost all of the existing walking robots created till now include in their mechanical structure a type of a walking mechanism. There are a variety of mechanisms developed during time from simple to more complex configurations used as support for generating walking movement. In order to design new concepts that are able to reproduce the walking characteristics of their biological inspiration models the type of walking of animals, reptiles, insects and humans is studied with attention (Doroftei and Adascalitei, 2012). A new attempt of a bio-inspired leg mechanism was conceived by Jansen (2007). The scalable design, reduced degree of mobility and low energy consumption are the main advantages that Jansen type of walking mechanism possess in comparison with other similar configurations (Nansai et al., 2013).

Studies regarding kinematics and dynamics of this configuration were done by Nansai et al. (2013), Giesbrecht and Wu Qiong (2012) and Moldovan (2013). Various optimization methods including genetic algorithms and adaptive network based fuzzy inference system are used in order to improve the existent link lengths and trajectory shape in Nansai et al. (2013). Studies for adapting the mechanism for climbing possibilities are conducted by Komoda and Wagatsuma (2011). The Jansen leg mechanism was studied by authors in Moldovan (2013), were the planar linkage was submitted to kinematic analysis and synthesis, $\mathrm{CAD}$ design and simulation.

Silva and Machado deal with a general overview regarding legged locomotion and the types of walking mechanisms used in the construction of walking robots (Silva and Machado, 2007). In order to develop new improved artificial walking systems a variety of leg configurations were studied and developed.

\footnotetext{
* To whom correspondence should be addressed
} 
Several configurations are based on well-known mechanisms (pantograph, Cebisev and parallel linkages) or specially designed (Jansen and Klann linkage).

Studies regarding motion characteristics described by a leg mechanism composed of a pantograph linkage combined with a Cebisev mechanism used for the design of biped robot with 1-DOF are conducted in LARM by Liang et al. (2008). The possibility for the mechanism to describe a trajectory shape similar to an ovoid curve is analyzed.

A new possibility for integrating a parallel mechanism in the configuration of a quadruped robot for assisting the people with disabilities is presented in Wang et al. (2013). The parallel leg mechanism has a modular configuration with 3 DOF formed by 3 serial parallel units.

Another variant of a walking mechanism is presented in Bałchanowski et al. (2009) specially implemented on a wheelchair based upon a four bar mechanism and combined with wheels.

The Klann linkage was studied and implemented in the structure of a new eight legged robot prototype in order to test its capabilities and the type of walking algorithm (Lokhande and Emche, 2013).

From the experiments conducted for determining the characteristics of walking (path curve for the characteristic point, step length and height) in the case of Jansen leg configuration, it has been concluded that for crossing over larger obstacles it will be necessary to increase the step height that this mechanism describes. To this end other solutions are proposed in order to modify the link length(s) through a supplementary actuator or to increase the number of leg mechanism DOF's and controlling the movement of the leg path.

This paper proposes a novel solution for improving the capability of walking mechanisms to cross over larger obstacles. From studies made for the trajectory described by the Jansen mechanism it appeared that the step height is not enough for crossing obstacles (Giesbretch and Wu Qiong, 2012). The novel solution consists in using of a 2 DOF 5-link belt mechanism that has the ability to reproduce multiple variants of path curves. By taking the Jansen mechanism path curve as the target trajectory and adapting the 5 link belt mechanism in order to describe similar trajectory with variable step height, the capabilities of it can be improved.

\section{Kinematic analysis of Jansen walking mechanism}

In order to compute the Jansen leg path it is necessary to perform the geometrical analysis. This study onvolves computing of the angular positions of each mechanism's elements and the coordinates of the coupler point $\mathrm{M}$ with its trajectory.

From the structural point of view the investigated mechanism is composed of eight bars connected through revolute joints and is assigned a single degree of freedom. It can be divided in three main closed loops of linkages: $A_{0} A B_{0} B$ (first four bar linkage), $A_{0} A E B_{0}$ (second four bar linkage) and final $B_{0} C D E$ (five bar linkage). The crank (2) accomplishes a complete rotation $(\varphi \in[0,2 \pi])$ while the end point $\mathrm{M}$ describes the trajectory presented in Fig.1. The origin of the coordinate system is set in $A_{0}$ fixed joint.

For computing the parametric coordinates of point $M$ through the whole walking cycle a mathematical model for geometrical analysis was developed. The equations were developed using the complex number method.

The equations of the closed loops are

$$
\begin{aligned}
& l_{2} \cdot e^{i \cdot \varphi}+l_{3} \cdot e^{i \cdot \vartheta_{1}}=\left(x_{B_{0}}+i \cdot y_{B_{0}}\right)+l_{4} \cdot e^{i \cdot \psi_{1}}, \\
& l_{2} \cdot e^{i \cdot \varphi}+l_{5} \cdot e^{i \cdot \vartheta_{2}}=\left(x_{B_{0}}+i \cdot y_{B_{0}}\right)+l_{6} \cdot e^{i \cdot \psi_{2}}, \\
& l_{41} \cdot e^{i \cdot\left(\alpha_{0}+\psi_{1}\right)}+l_{7} \cdot e^{i \cdot \gamma}=l_{6} \cdot e^{i \cdot \psi_{2}}+l_{8} \cdot e^{i \cdot \delta} .
\end{aligned}
$$

where $x_{B_{0}}, y_{B_{0}}$ are known coordinates. 


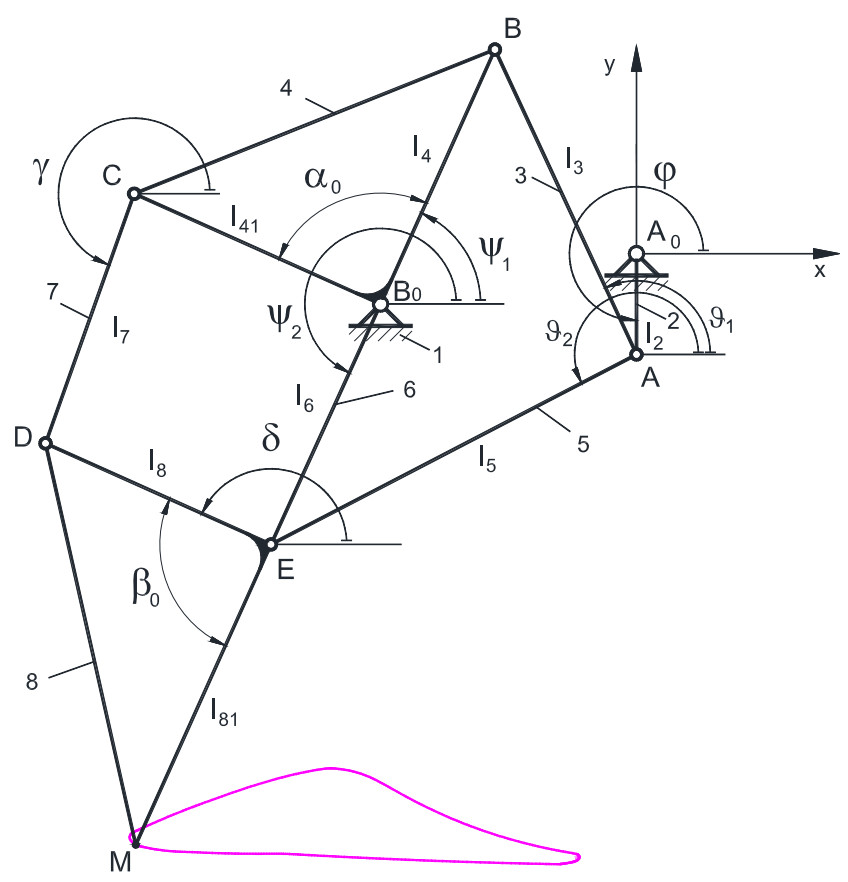

Fig.1. Jansen mechanism.

By separating of term which contains the angle $\left(\vartheta_{1}\right.$ or $\left.\vartheta_{2}\right)$ in the first, or, second Eqs $(2.1)$, respectively, and through multiplication with its complex conjugate relationship an equation in the form below results

$$
A_{i}(\varphi) \cdot \cos \psi_{i}+B_{i}(\varphi) \cdot \sin \psi_{i}+C_{i}(\varphi)=0, \quad i=1,2,
$$

where

$$
\begin{aligned}
& A_{l}(\varphi)=2 l_{4} x_{B_{0}}-2 l_{2} l_{4} \cdot \cos \varphi, \quad B_{l}(\varphi)=2 l_{4} y_{B_{0}}+2 l_{2} l_{4} \cdot \sin \varphi, \\
& C_{l}(\varphi)=x_{B_{0}}^{2}+y_{B_{0}}^{2}+l_{2}^{2}-l_{3}^{2}+l_{4}^{2}-2 l_{2} x_{B_{0}} \cdot \cos \varphi-2 l_{2} y_{B_{0}} \cdot \sin \varphi, \\
& A_{2}(\varphi)=2 l_{6} x_{B_{0}}-2 l_{2} l_{6} \cdot \cos \varphi, \quad B_{2}(\varphi)=2 l_{6} y_{B_{0}}+2 l_{2} l_{6} \cdot \sin \varphi, \\
& C_{2}(\varphi)=x_{B_{0}}^{2}+y_{B_{0}}^{2}+l_{2}^{2}-l_{5}^{2}+l_{6}^{2}-2 l_{2} x_{B_{0}} \cdot \cos \varphi-2 l_{2} y_{B_{0}} \cdot \sin \varphi,
\end{aligned}
$$

and next from Eq.(2.2) we get the expression for position angle $\psi_{i}(i=1,2)$

$$
\psi_{i}(\varphi)=2 \cdot a \tan \frac{B_{i}(\varphi)-\sqrt{A_{i}(\varphi)^{2}+B_{i}(\varphi)^{2}-C_{i}(\varphi)^{2}}}{A_{i}(\varphi)-C_{i}(\varphi)} .
$$

A similar procedure is applied for the third closed loop for computing the position angles $(\delta)$ 


$$
\begin{aligned}
& A_{3}(\varphi) \cdot \cos \delta+B_{3}(\varphi) \cdot \cos \delta+C_{3}(\varphi)=0, \\
& \delta(\varphi)=2 \cdot a \tan \frac{B_{3}(\varphi)-\sqrt{A_{3}(\varphi)^{2}+B_{3}(\varphi)^{2}-C_{3}(\varphi)^{2}}}{A_{3}(\varphi)-C_{3}(\varphi)} .
\end{aligned}
$$

where

$$
\begin{aligned}
& A_{3}(\varphi)=2 l_{6} l_{8} \cdot \cos \psi_{2}-2 l_{41} l_{8} \cdot \cos \left(\psi_{1}+\alpha_{0}\right), \\
& B_{3}(\varphi)=-2 l_{6} l_{8} \cdot \sin \psi_{2}+2 l_{41} l_{8} \cdot \sin \left(\psi_{1}+\alpha_{0}\right), \\
& C_{3}(\varphi)=l_{41}^{2}+l_{6}^{2}-l_{7}^{2}+l_{8}^{2}+2 l_{6} l_{41} \cdot \cos \left(\psi_{1}+\alpha_{0}\right) .
\end{aligned}
$$

The parametric coordinates of the point $\mathrm{M}$ are described through the following relations

$$
\begin{aligned}
& x_{M}+i \cdot y_{M}=x_{B_{0}}+i \cdot y_{B_{0}}+l_{6} \cdot e^{i \cdot \psi_{2}(\varphi)}+l_{81} \cdot e^{i \cdot\left(\delta(\varphi)+\beta_{0}\right)}, \\
& \left\{\begin{array}{l}
x_{M}(\varphi)=x_{B_{0}}+l_{6} \cdot \cos \psi_{2}(\varphi)+l_{81} \cdot \cos \left(\delta(\varphi)+\beta_{0}\right), \\
y_{M}(\varphi)=y_{B_{0}}+l_{6} \cdot \sin \psi_{2}(\varphi)+l_{81} \cdot \sin \left(\delta(\varphi)+\beta_{0}\right) .
\end{array}\right.
\end{aligned}
$$

After implementing these equations in a MathCad program based on input data characterized by the link lengths in $\mathrm{mm}\left(l_{2}=15, l_{3}=50, l_{4}=41.5, l_{41}=40.1, l_{5}=61.9, l_{6}=39.3, l_{7}=39.4, l_{8}=36.7, l_{81}=49, \alpha_{0}=90^{\circ}\right.$, $\left.\beta_{0}=90^{\circ}\right)$, the coordinates for $A_{0}\left(x_{A 0}=0, y_{A 0}=0\right), B_{0}\left(x_{B 0}=-38, y_{B 0}=-7.5\right)$, input values of the angle $\varphi$ from the crankshaft (2) and position of $B_{0}$ fixed couple, the coordinates of the end point $\mathrm{M}$ were calculated. The trajectory shape described by the leg mechanism based on the coordinates achieved is presented in Fig.2.

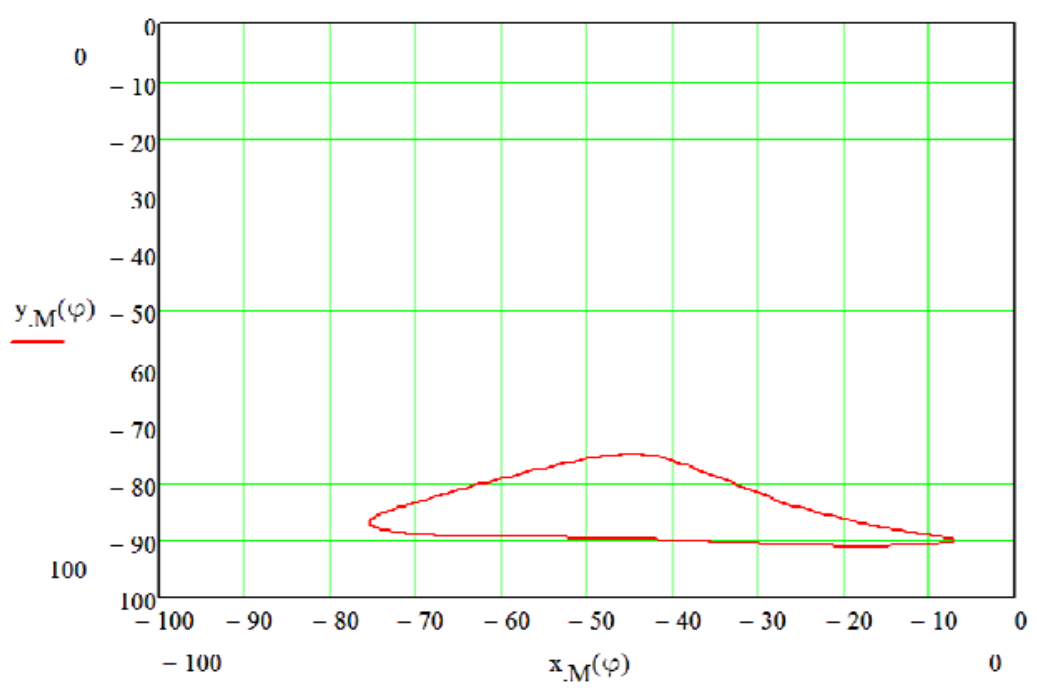

Fig.2. View of Jansen leg mechanism's trajectory. 


\section{Kinematic analysis of the 5-link belt mechanism}

A special form of a planar mechanism with 2 DOF called 5-link belt mechanism is proposed to be used as a leg mechanism configuration in order to increase the step height.

This type of mechanism can be used for generating special trajectories. It has a variable link length, based on a belt as a flexible and inextensible element that is enveloped or developed on a circular wheel (see Fig.3).

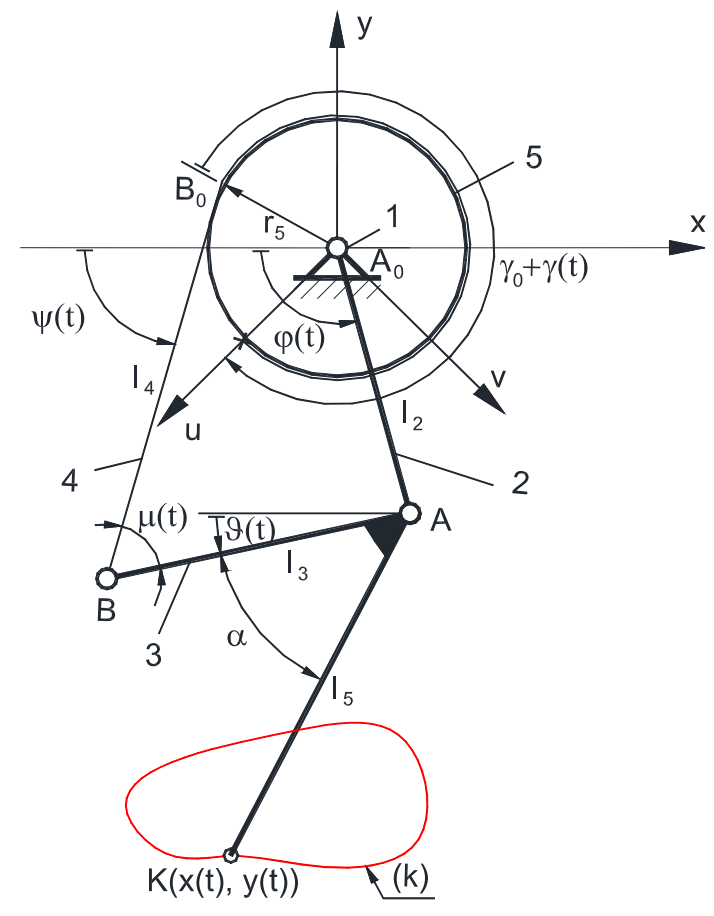

Fig.3. Kinematic schema of 5-link belt mechanism.

The link (2) and the circular wheel (2.5) are driving elements. The trajectory for the characteristic point $K$ is given in a parameterized form, with $t \in[0,1]$

$$
K=x(t)+i \cdot y(t)
$$

and the same trajectory (2.1) can be described with the coupler point $K$ of the 5-link belt mechanism by the relationship, written in complex numbers

$$
K=l_{2} \cdot e^{i \cdot \varphi(t)}+l_{5} \cdot e^{i \cdot(\alpha+\vartheta(t))} .
$$

From Eqs (3.1) and (3.2) equalized and by eliminating the exponent $\alpha+\vartheta(t)$ we get

$$
\varphi(t)=2 \cdot \arctan \frac{B(t) \pm \sqrt{A^{2}(t)+B^{2}(t)-C^{2}(t)}}{A(t)-C(t)}
$$

where 


$$
A(t)=2 l_{2} \cdot x(t) \quad B(t)=2 l_{2} \cdot y(t) \quad C(t)=l_{5}^{2}-x^{2}(t)-y^{2}(t)-l_{2}^{2} .
$$

In an analog procedure the positional angle of the coupler (2.3) gives

$$
\vartheta(t)=\varphi(t)-\alpha+\arccos \left(\frac{x^{2}(t)+y^{2}(t)-l_{2}^{2}-l_{5}^{2}}{2 l_{2} l_{5}}\right) .
$$

Further we present the calculus of the drive angle $\theta(t)$ of the wheel and the calculus of the wheel angle $\gamma(t)$ in the mobile system of the wheel and belt angle $\psi(t)$ in the frame system

$$
\theta(t)=\psi(t)-\gamma(t)
$$

where

$$
\begin{aligned}
& \gamma(t)=\frac{l-\sqrt{l_{2}^{2}+l_{3}^{2}-r_{5}^{2}+2 l_{2} l_{3} \cdot \cos (\varphi(t)-\vartheta(t))}}{r_{5}}-\gamma_{0}, \\
& \psi(t)=2 \arctan \frac{r_{5} \mp \sqrt{r_{5}^{2}+l_{4}(t)^{2}-\left(l_{2} \cdot \cos \varphi(t)+l_{3} \cdot \cos \vartheta(t)\right)^{2}}}{l_{4}(t)+l_{2} \cdot \cos \varphi(t)+l_{3} \cdot \cos \vartheta(t)} .
\end{aligned}
$$

with: $l$ - the total length of the belt, $r_{5}$ - the radius of the circular wheel and $\gamma_{0}$ the initial enveloped belt angle for $t=0$.

In order to avoid the singularities, it is necessary to have the transmission angle always positive and $\mu(t)>\mu_{\min }$, where

$$
\mu(t)=\psi(t)-\vartheta(t)
$$

\section{Numerical example for the novel of the 5-link belt mechanism}

Starting with the parameterization for the Jansen mechanism path curve obtained through the parameterized coordinates (2.8) that lead to dependency of parameter $t \in[0,1]$ instead of $\varphi$, the trajectory is divided into seven sub-curves. For each sub-curve an interpolation function is applied based on a second order spline. This method is chosen in order to have a unitary form for each polynomial expression corresponding to the sub-curve and to avoid the global forms of interpolation that usually do not converge. The new parametric equations obtained will be further used for generating new trajectories which are different from the initial one.

The parametric equations for the curve are expressed depending of the parameter $t$ as in

$$
\begin{aligned}
& x_{M, i}(t)=x_{i}+m_{x i}\left(t-t_{i}\right)+a_{x i}\left(t-t_{i}\right)^{2}, \quad y_{M, i}(t)=y_{i}+m_{y i}\left(t-t_{i}\right)+a_{y i}\left(t-t_{i}\right)^{2}, \\
& m_{x i}+2 \cdot a_{x i} \cdot\left(t_{i+1}-t_{i}\right), \quad m_{y i}+2 \cdot a_{y i} \cdot\left(t_{i+1}-t_{i}\right),
\end{aligned}
$$




$$
a_{x i}=\frac{x_{i+1}-x_{i}}{\left(t_{i+1}-t_{i}\right)^{2}}-\frac{m_{x i}}{t_{i+1}-t_{i}}, \quad a_{y i}=\frac{y_{i+1}-y_{i}}{\left(t_{i+1}-t_{i}\right)^{2}}-\frac{m_{y i}}{t_{i+1}-t_{i}}
$$

where $i$ represents the interpolation range, $m_{x i}, m_{y i}$ are the slope of the segment curve and the second order coefficients $a_{x i}, a_{y i}$.

The interpolation values and input geometrical data used in the following example for the 5-link belt mechanism are presented in Tab. 1 and Tab. 2 .

Table 1. Interpolation values for the Jansen leg trajectory.

\begin{tabular}{|c|c|c|c|c|c|c|c|c|}
\hline$m_{x 1}$ & 103.487 & & $m_{y 1}$ & -7.874 & \multicolumn{3}{|c|}{} \\
\hline$i$ & 1 & 2 & 3 & 4 & 5 & 6 & 7 & 8 \\
\hline$t_{i}$ & 0 & 0.389 & 0.553 & 0.639 & 0.764 & 0.833 & 0.958 & 1 \\
\hline$x_{i}$ & -74.363 & -26.179 & -7.636 & -10.581 & -39.419 & -59.112 & -75.319 & -74.363 \\
\hline$y_{i}$ & -87.965 & -90.811 & -90.395 & -85.984 & -46.329 & -45.890 & -86.849 & -87.965 \\
\hline
\end{tabular}

Table 2. Input geometric data of the 5-link belt mechanisms.

\begin{tabular}{|l|c|l|l|l|l|l|l|}
\hline $\begin{array}{l}\text { Frame } \\
\text { length }\end{array}$ & $l_{1}=0 \mathrm{~mm}$ & $\begin{array}{l}\text { Coupler } \\
\text { length }\end{array}$ & $l_{3}=10 \mathrm{~mm}$ & Belt length & $l=650 \mathrm{~mm}$ & $\begin{array}{l}\text { Coupler } \\
\text { angle }\end{array}$ & $\alpha^{\circ}=135^{\circ}$ \\
\hline $\begin{array}{l}\text { Crank } \\
\text { length }\end{array}$ & $l_{2}=55 \mathrm{~mm}$ & $\begin{array}{l}\text { Coupler } \\
\text { length }\end{array}$ & $l_{5}=50 \mathrm{~mm}$ & $\begin{array}{l}\text { Wheel } \\
\text { radius }\end{array}$ & $r_{5}=20 \mathrm{~mm}$ & \multicolumn{2}{|c}{} \\
\hline
\end{tabular}

After introducing and computing the mathematical relations for the 5-link belt mechanism with the chosen parameters in different positions corresponding to the control points from the given curve we obtained the drive angles $\theta(t), \varphi(t)$, wheel angle $\gamma(t)$, belt angles $\psi(t)$ and transmission angles $\mu(t)$ presented in Figs 4,5 and 6. This operations were done for three different paths with various heights $(h=30,42,55 \mathrm{~mm})$.

a

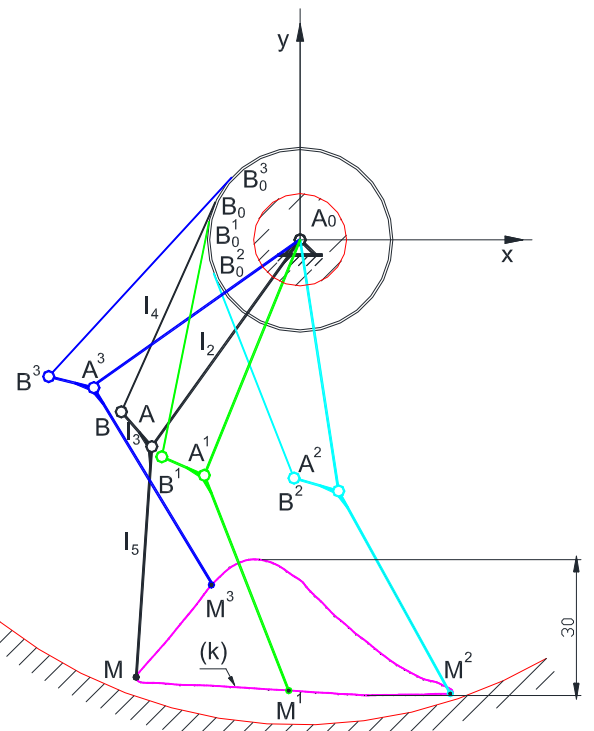

b

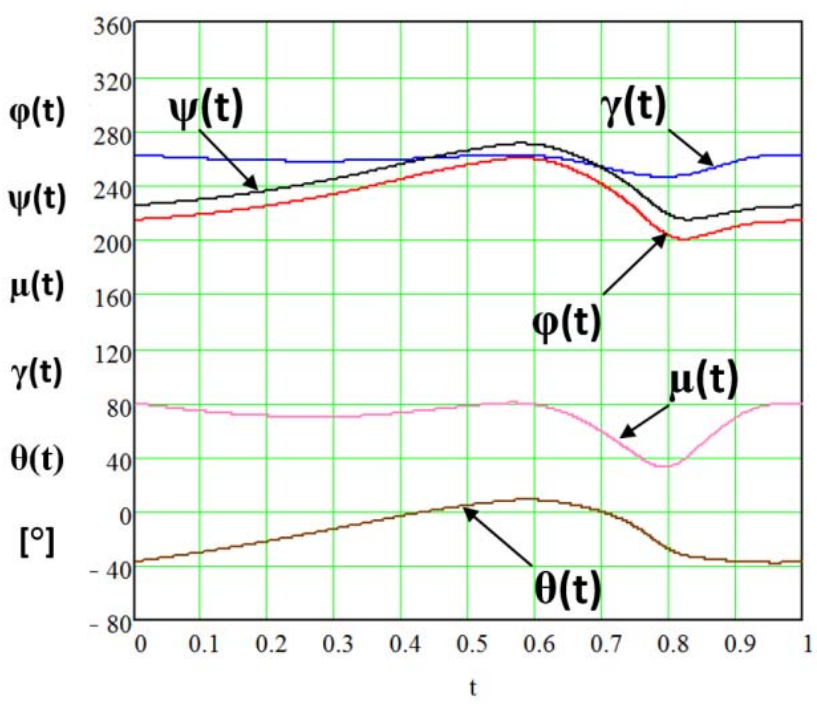

Fig.4. a) The 5-link belt mechanism generating a trajectory with $h=30 \mathrm{~mm}$; b) The drive angles $\varphi(t), \theta(t)$, belt angles $\psi(t)$, wheel angle $\gamma(t)$ and transmission angles $\mu(t)$. 


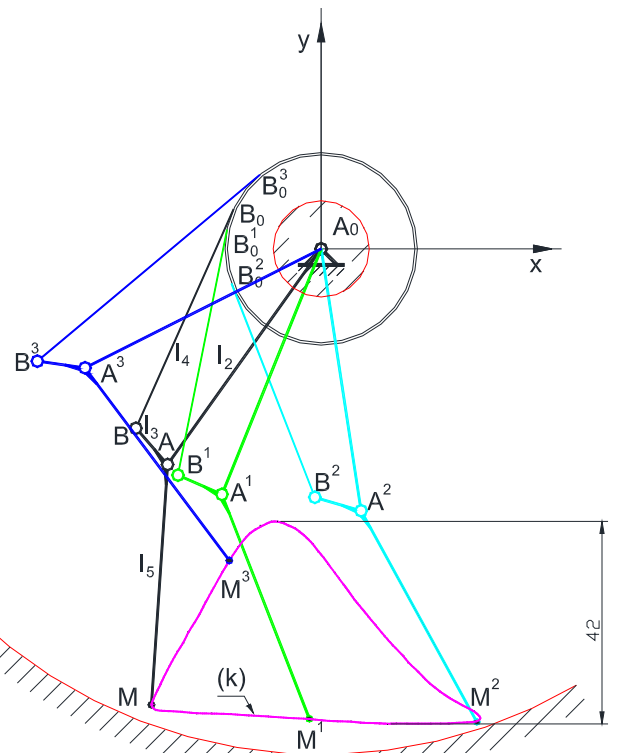

b

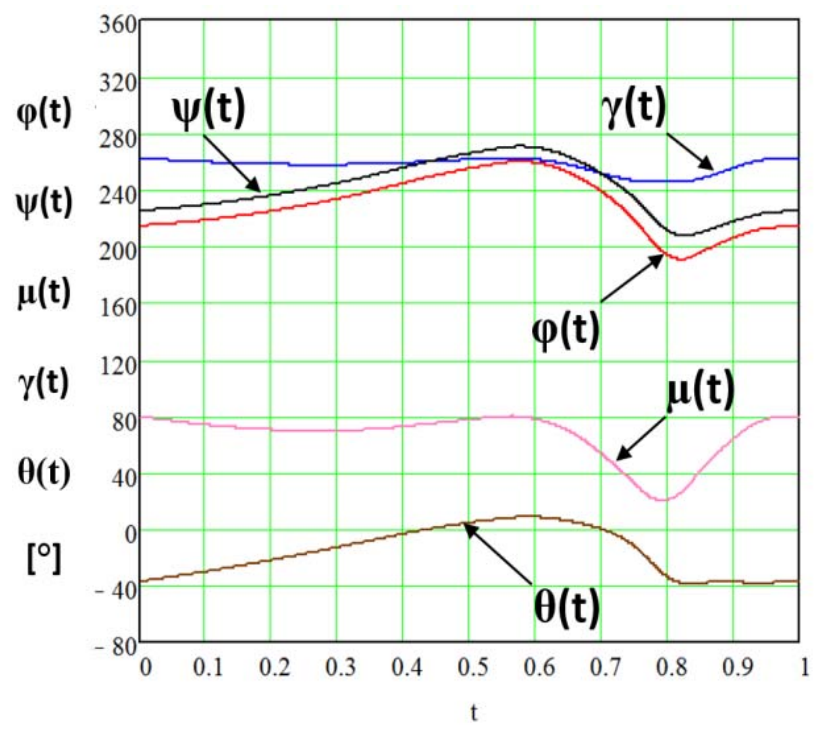

Fig.5. a) The 5 link belt mechanism generating a trajectory with $h=42 \mathrm{~mm}$; b) The drive angles $\varphi(t), \theta(t)$, belt angles $\psi(t)$, wheel angle $\gamma(t)$ and transmission angles $\mu(t)$.

a

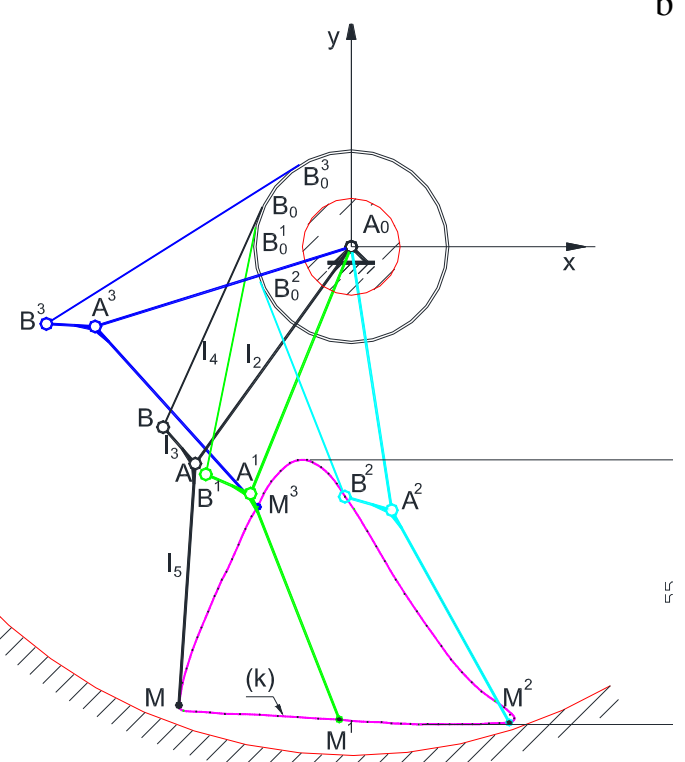

b

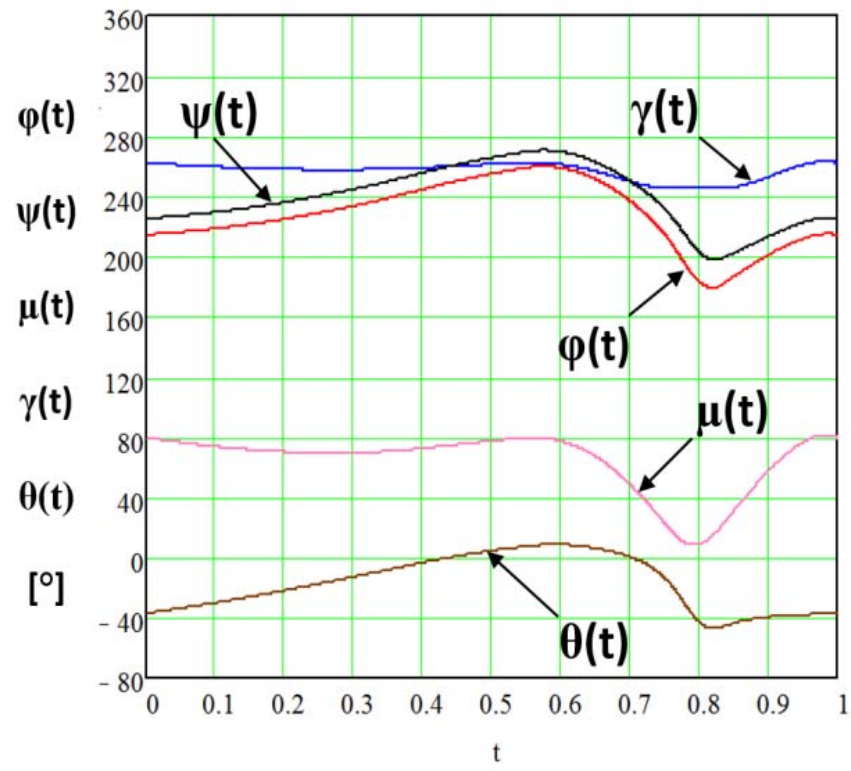

Fig.6. The 5 link belt mechanism generating a trajectory with $h=55 \mathrm{~mm}$; b) The drive angles $\varphi(t), \theta(t)$, belt angles $\psi(t)$, wheel angle $\gamma(t)$ and transmission angles $\mu(t)$.

\section{Results and conclusions}

The proposed novel leg mechanism solution using a 5-link belt mechanism is viable because it can properly reproduce the Jansen characteristic point trajectory and the control functions for the driving 
elements can be easily implemented. As shown in Figs 4, 5 and 6 the controlled rotation angles do not have jumps in their variation towards the parameter $t$.

Also the transmission angle is in a positive range of values, which indicates that singularities are avoided throughout the entire control range.

It can be stated that, the novel mechanism structure through the control of the actuators (driving elements) can be adapted to follow both the Jansen trajectory and similar trajectories with different step heights. The step height can be improved in order to avoid different obstacles.

\section{Nomenclature}

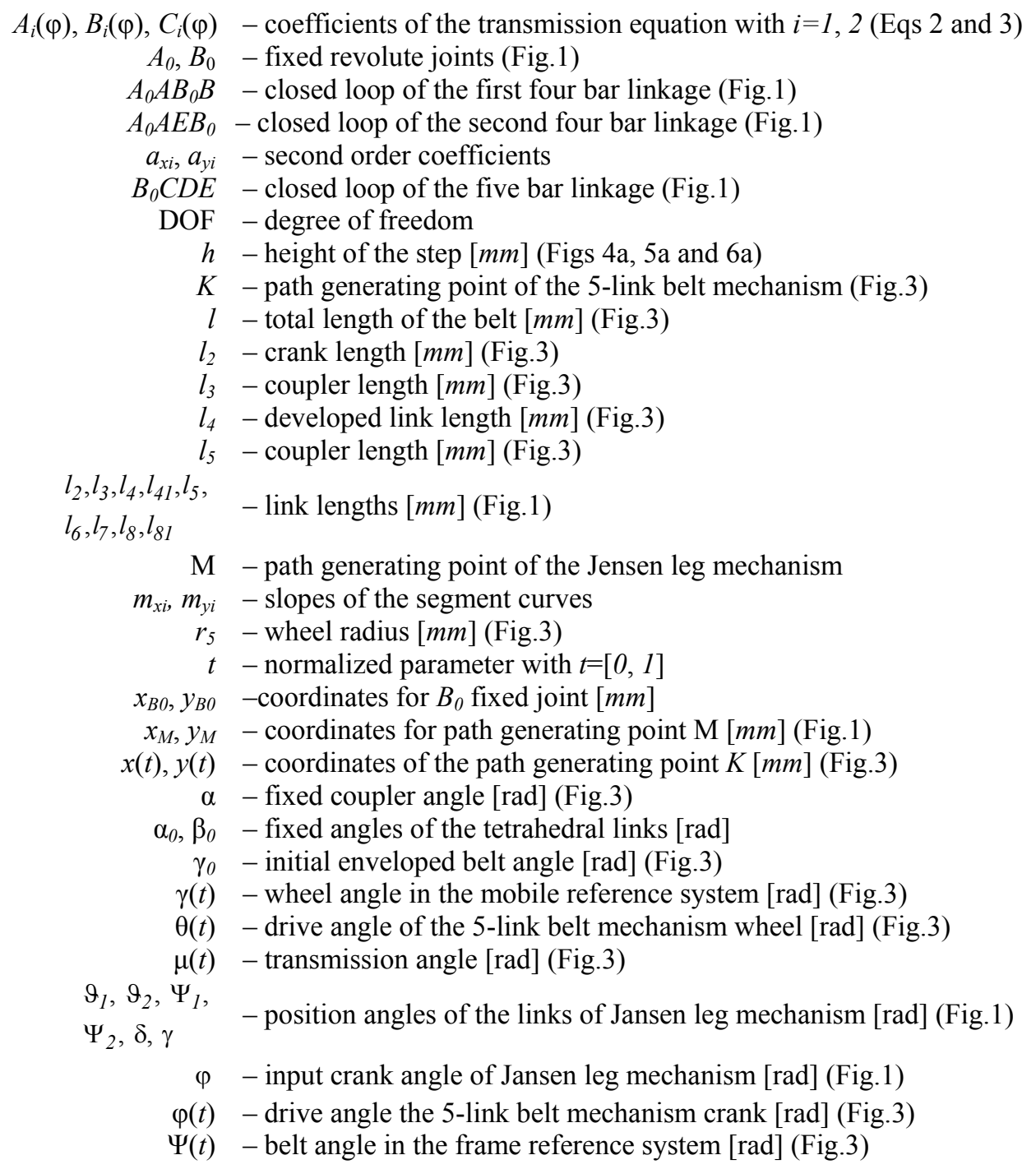

\section{References}

Bałchanowski J., Szrek J. and Wudarczyk S. (2009): Wheelchair mechanism for negotiating obstacles. - The Archive of Mechanical Engineering, No.3, vol.LVI, pp.251-261.

Doroftei I. and Adascalitei F. (2012): A hexapod walking micro-robot with compliant legs. - Applied Mechanics and Materials, vol.162, pp.234-241. 
Giesbrecht D. and Wu Qiong C. (2012): Design and optimization of an eight-bar legged walking mechanism imitating a kinetic sculpture. - "Wind Beast", Transactions of the Canadian Society for Mechanical Engineering, vol.36, No.4, pp.343-355.

Jansen T. (2007): The Great Pretender. - 010 Publishers, Rotterdam.

Komoda K. and Wagatsuma H. (2011): A study of availability and extensibility of Theo Jansen mechanism toward climbing over bumps. - The 21st Annual Conference of the Japanese Neural Network Society, December, pp.28.

Liang C., Ceccarelli M. and Takeda Y. (2008): Operation Analysis of a One-DOF Pantograph Leg Mechanisms. Proceedings of the RAAD 2008, 17th International Workshop on Robotics in Alpe-Adria-Danube Region, September 15-17, Ancona, Italy.

Lokhande N.G. and Emche V.B. (2013): Mechanical Spider by Using Klann Mechanism. - International Journal of Mechanical Engineering and Computer Applications, vol.1, No.5, Special Issue, pp.13-16.

Lovasz E.-C., Perju D., Modler K.-H., Gruescu C.M., Mărgineanu D., Moldovan C.E. and Pop C. (2014): Path Generating Belt Mechanisms as Kinematic Chains for Mechatronic Applications. - EUCOMES, Guimarães Portugal, 2014.

Moldovan F. (2013): Cercetari privind analiza si sinteza unui mecanism cu bare pentru constructia unui robot mobil pasitor (Research regarding analysis and synthesis of a bar mechanism for constructing a walking robot). - $\mathrm{PhD}$ thesis, Timisoara.

Nansai S., Rajesh Elarab M. and Iwasea M. (2013): Dynamic analysis and modelling of Jansen mechanism. - Procedia Engineering 64, pp.1562-1571.

Silva F.M. and Machado T.J.A. (2007): A Historical perspective of legged robots. - Journal of Vibration and Control September ,vol.13, No.9-10, pp.1447-1486.

Wang H., Sang L., Hu X., Zhang D. and Yu H. (2013): Kinematics and dynamics analysis of a quadruped walking robot with parallel leg mechanism. - Chinese Journal of Mechanical Engineering, vol.26, No.5, pp.881-891.

Received: June 4, 2014

Revised: October 1, 2014 